\title{
Erratum
}

Arch Pharm Res Vol 28, No 8, 877-884 (2005)

\section{Structure and Antiinflammatory Activity Relationships of Wogonin Derivatives}

Jinhee Jang, Hyun Pyo Kim, and Haeil Park

College of Pharmacy, Kangwon National University, Chuncheon 200-701, Korea

One of author named Kwan-Seog Sin should be added as shown in the following :

Jinhee Jang, Kwan-Seog Sin, Hyun Pyo Kim, and Haeil Park

College of Pharmacy, Kangwon National University, Chuncheon 200-701, Korea 